\title{
Tolerability of Isoniazid Preventive Therapy in an HIV-Infected Cohort of Paediatric and Adolescent Patients on Antiretroviral Therapy from a Resource-Limited Setting: A Retrospective Cohort Study
}

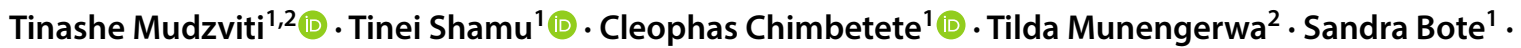 \\ Margaret Pascoe ${ }^{1}$
}

Published online: 13 February 2019

(c) The Author(s) 2019

\begin{abstract}
Background Treating patients with latent tuberculosis infection (LTBI) to prevent development of active disease is an essential strategy for eliminating TB. There are concerns regarding the use of isoniazid due to the potential for hepatotoxicity. This study was conducted to determine the incidence of adverse hepatic events after isoniazid preventive therapy (IPT) commencement in a cohort of HIV-infected paediatric and adolescent patients on antiretroviral therapy (ART).

Methods This was a retrospective records review, using data from HIV-infected paediatric and adolescent patients collected during routine clinical visits at Newlands Clinic, Harare, Zimbabwe. Patients included in the analysis had commenced IPT between January 2014 and June 2015 (inclusive) whilst receiving ART. A survival analysis was conducted for the period that participants were receiving IPT with end-points defined by grade 3 or grade 4 elevations in alanine aminotransferase (ALT) levels.

Results Data from 438 patients commenced on IPT were analysed; 202 (46.1\%) of them were female. The median age at IPT commencement was 10 (IQR $=7-12$ ) years. Twenty-eight patients developed grade 3 or 4 elevations in ALT. Concomitant use of nevirapine as part of an ART regimen was the only factor that showed a statistically significant association with ALT elevation [relative risk (RR): 2.7; confidence interval (CI): 1.2-6.3, $p=0.012$ ] compared with those not receiving nevirapine. The incidence of grade 3 or 4 elevations in ALT was 31.5/100 person-years (CI 20.9-45.5).

Conclusion The incidence of IPT-associated ALT elevations was high in this population. We recommend vigilant monitoring of liver enzymes for patients receiving IPT, especially in patients concomitantly receiving nevirapine.
\end{abstract}

\section{Key Points}

Use of isoniazid in the prevention of tuberculosis has the potential to cause hepatotoxicity in children receiving nevirapine.

Children receiving isoniazid preventive therapy should be routinely monitored for liver function.

Tinashe Mudzviti

tinashem@newlandsclinic.org.zw

1 Newlands Clinic, 56 Enterprise Road, Highlands, Harare, Zimbabwe

2 School of Pharmacy, University of Zimbabwe, P.O. Box MP167, Mount Pleasant, Harare, Zimbabwe

\section{Introduction}

Treating patients with latent tuberculosis infection (LTBI) to prevent the development of active disease is an essential strategy for eliminating tuberculosis (TB). In 2014, globally 9.6 million people were estimated to have fallen ill with TB, amongst which children constituted 1.0 million of the total. The actual burden is likely to be higher, because diagnosing $\mathrm{TB}$ in children is challenging and is a low priority in lowresource settings [1]. In general, isoniazid therapy is recommended for 6-12 months [2], with an efficacy of approximately $90 \%$ among patients who complete treatment [3]. However, there are concerns regarding the use of isoniazid due to the potential for hepatotoxicity, ranging from asymptomatic elevation of liver enzymes to severe hepatitis [4]. Isoniazid is a potentially hepatotoxic medicine and its use as an agent for IPT could result in liver toxicities. Isoniazid 
is an inhibitor of CYP 2B6 and has the potential to increase the concentrations of concurrently administered medicines metabolized by this enzyme.

The Thibela TB study, which was a cluster randomized trial of community-wide isoniazid preventive therapy (IPT) in South Africa, described adverse events with IPT in a study population of around 80,000 participants [5]. In this study the investigators concluded that the risk of adverse events as a result of IPT, particularly hepatotoxicity, was very low in this population $(0.07 \%)$. Several other studies have also shown that there are low rates of hepatotoxicity when IPT is administered to HIV-infected individuals [3, 4, 6, 7]. Seven pilot sites in Zimbabwe implemented 6 months of IPT from 31 December 2012 to 1 March 2013 in PLHIV [8]. Of the 578 participants that received IPT in this pilot study, only $8(7.2 \%)$ patients were documented as not completing the IPT course due to the development of toxicity. However, it is important to note that Takarinda et al. [8] report a 59.8\% loss to follow-up rate, which is quite high. Whilst Takarinda et al. report an $80 \%$ completion rate of IPT under routine conditions, there is a need to examine further the impact of toxicities on IPT discontinuation. Due to the high rates of both HIV and TB in sub-Saharan African countries including Zimbabwe versus Western Countries there is a need to evaluate toxicities of isoniazid in HIV-infected patients. In a systematic review evaluating IPT in HIV-infected children, clinical adverse events were similar between the isoniazid prophylaxis and placebo groups, both in children not on ART and those on ART. Laboratory adverse events were similar between the isoniazid prophylaxis and placebo groups, both in children not on ART and those on ART [9]. In a secondary analysis of Zar et al.'s data, Le Roux et al. showed that 16 (5.4\%) out of 297 children developed severe liver injury while receiving isoniazid, but that only $1.7 \%$ of these cases were related to isoniazid prophylaxis $[7,9,10]$.

Nevirapine, which is also a potentially hepatotoxic medicine, is metabolized by the enzyme CYP 2B6. Therefore, co-administration of nevirapine with isoniazid might expose patients to a drug-drug interaction that is hepatotoxic in nature [11]. Currently available limited data advises that the concurrent administration of isoniazid with nevirapine is relatively safe $[12,13]$. In a brief report by Decloedt et al., an evaluation of the pharmacokinetics of nevirapine when given with isoniazid showed that co-administration was associated with a $24 \%$ increase in median nevirapine area under the curve within the first $12 \mathrm{~h}$. This result was, however, not statistically significant and the sample size used in this study was too small to establish the effect of slow and fast isoniazid acetylators on nevirapine as it is possible that the inhibiting effect of isoniazid in slow acetylators may be more pronounced [14].

The objectives of the study were to estimate the incidence of adverse hepatic events as assessed by alanine aminotransferase (ALT) elevation after IPT commencement in a cohort of HIV-infected paediatric and adolescent patients on antiretroviral therapy (ART). We also set out to evaluate the severity of the hepatic adverse events in the population of paediatric and adolescent population concurrently receiving ART with isoniazid. Finally, we sought to describe which demographic and patient treatment factors were associated with hepatic events and discontinuations of IPT (age, gender and concurrent nevirapine use).

\section{Methods}

This was a retrospective cohort study in which a records review was conducted using existing data captured during routine clinical visits at Newlands Clinic, Harare Zimbabwe. The details of the clinical operations at Newlands Clinic are described elsewhere [15].

\subsection{Data Collection}

Baseline was defined as the day on which IPT was commenced. Newlands Clinic maintains an electronic medical database where all patient data are stored. The database contains patient demographic information, procedures carried out on the patient at each clinic visit, diagnoses made, medication prescribed, and medication dispensed. All laboratory tests and investigations conducted are also recorded in the database for the respective patients. We included all eligible participants in the database who received IPT between January 2014 and May 2015. De-identified data were extracted from the database onto an excel spreadsheet. The variables extracted were patient sex; age; medications taken whilst receiving IPT including duration of use, reason for discontinuation; ALT test results at baseline and during IPT use; baseline CD4 count; baseline viral load count; and any history of TB therapy. Excluded from commencing IPT were those patients who presented with signs and symptoms suggestive of TB, patients who were already on TB treatment, patients with signs of active liver disease, and patients with a history of isoniazid-induced hepatitis or heavy alcohol use. Exposure status was defined as receipt of isoniazid for treatment of LTBI whilst receiving ART. ART use in the participants was defined as continued receipt of antiretroviral medicines for a period of at least 3 months. A baseline viral load result was defined as the closest recorded result within 3 months of the date when IPT was commenced. A baseline CD4 count result was defined as the closest recorded result within 6 months of the date when IPT was commenced. Patients were followed up for the 24 weeks that that they were receiving IPT or if they reached a study endpoint earlier. 
ALT levels were used to evaluate adverse hepatic events. Normal ALT levels were regarded as those within the range 0-41 international U/L. Hepatotoxicity was categorized by ALT elevations, with grade 1 and 2 being ALT levels between 42 and $200 \mathrm{U} / \mathrm{L}$, and grade 3 and 4 being $>200$ U/L [16]. Patients who had grade 1 and 2 elevations in ALT would continue IPT whilst being monitored and if they developed grade 3 or 4 elevations, IPT was discontinued.

\subsection{Ethical Considerations}

Permission to carry out this study was granted by the Newlands Clinic Scientific Committee and the Medical Research Council of Zimbabwe (MRCZ/E/139).

\subsection{Statistical Analysis}

Data were analysed using Stata (ver. 12) for descriptive statistics and measures of association. Descriptive statistics including frequencies, proportions, medians and interquartile ranges were used to describe the baseline characteristics and ALT outcomes. Cox proportional hazards were used to determine risk factors for grade 3 or 4 hepatotoxicity. Time zero of follow-up was the day of IPT commencement with endpoints being IPT completion, discontinuation or development of grade 3 or 4 hepatotoxicity. Kaplan-Meir curves were constructed to estimate survival rates while on IPT with end-points being defined by grade 3 or 4 elevations in ALT levels. A univariate analysis was conducted to identify if any of the potentially hepatotoxic antiretroviral medicines had an association with elevations in ALT. The ARVs that were included in the univariate analysis were nevirapine, efavirenz, lopinavir and atazanavir. A multivariate analysis was then conducted to evaluate whether those factors that were significant in the univariate analysis would remain significant.

\section{Results}

Five hundred and seventy-one patients were commenced on IPT between January 2014 and May 2015. 458 of these patients had complete ALT laboratory data that could be analysed. Data for 20 patients were excluded from this analysis based on having pre-existing grade 1 elevations in ALT. Figure 1 displays the enrolment and outcomes observed.

The characteristics of the participants in this study are shown in Table 1. The median age at IPT commencement was 10 [interquartile range (IQR) $=7-12$ ] years. The majority of participants had nevirapine as the third ARV medicine as dictated by the Zimbabwe National ART guidelines. Nevirapine in combination with two nucleoside reverse transcriptase inhibitors was the preferred first-line regimen [17].

Severity of ALT elevations were categorized as grade 1 and 2 or grade 3 and 4 . The median time to developing ALT

Table 1 Patient characteristics $(N=438)$

\begin{tabular}{ll}
\hline Variable & Statistic \\
\hline Sex & \\
$\quad$ Female, $n(\%)$ & $202(46.1)$ \\
Age at IPT commencement (years) & $10.0(7-12)$ \\
Median (IQR) & $1-20$ \\
Range & \\
Concomitant nevirapine use, $n(\%)$ & $229(52.3)$ \\
Yes & $209(47.7)$ \\
No & \\
\hline
\end{tabular}

$I P T$ isoniazid preventive therapy, IQR interquartile range

Fig. 1 Enrolment and outcomes. IPT isoniazid preventive therapy, $A L T$ alanine aminotransferase, $A R T$ antiretroviral treatment

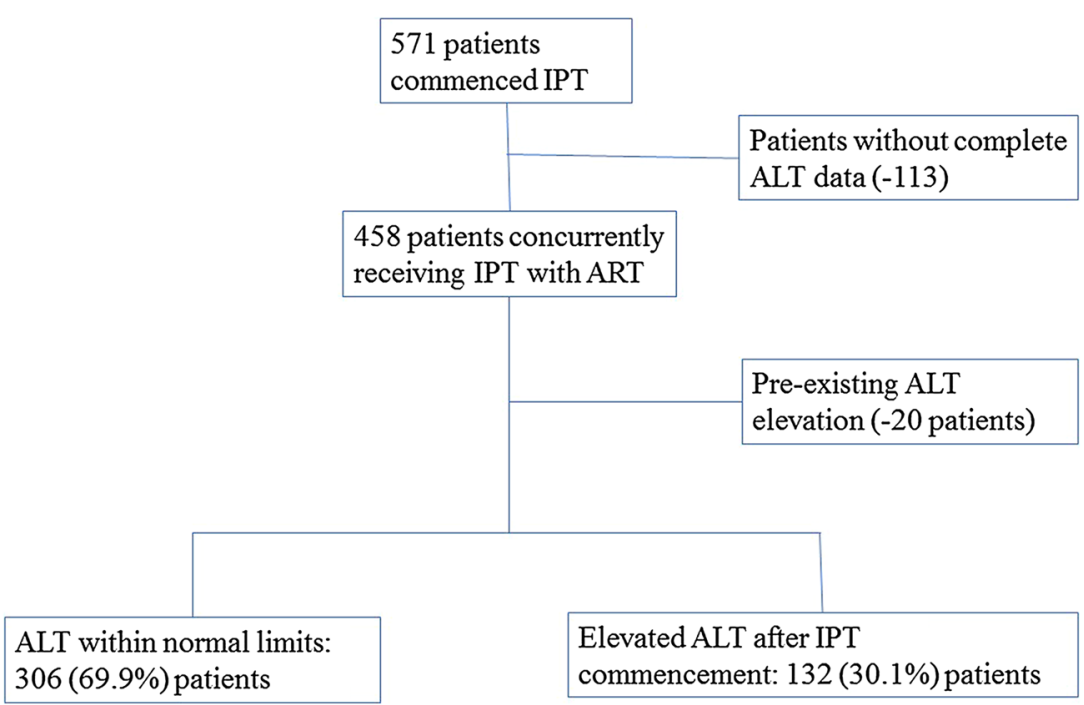


Table 2 Severity of ALT elevations $(n=132)$

\begin{tabular}{ll}
\hline Variable & Statistic \\
\hline Grade 1 and 2 ALT elevations, $n(\%)$ & $104(79)$ \\
Median ALT (U/L) (IQR) & $66(48-103)$ \\
Median time to grade 1 or 2 elevation, weeks & $12(8-19)$ \\
$\quad$ (IQR) & \\
Grade 3 and 4 ALT elevations, $n(\%)$ & $28(21)$ \\
Median ALT (U/L) (IQR) & $335(257-420)$ \\
Median time to grade 3 or 4 elevation, weeks & $12(9-16)$ \\
(IQR) & \\
Incidence & $31.5 / 100$ person- \\
& years $(\mathrm{CI}$ \\
& $20.9-45.5)$ \\
\hline
\end{tabular}

$A L T$ alanine aminotransferase, $I Q R$ interquartile range

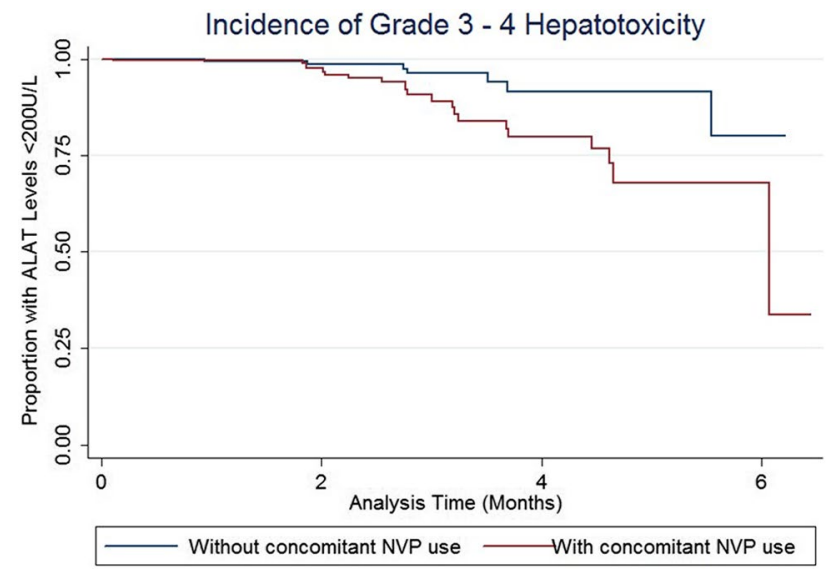

Fig. 2 Kaplan-Meier curves demonstrating incidence of grade 3 or 4 ALT elevations in the 438 participants during IPT. ALT alanine aminotransferase, $N V P$ nevirapine

elevation was 12 weeks regardless of the severity. Table 2 shows a description of the distribution of ALT elevations.

In a Cox proportional hazard model, age, gender and individual ARV use were analysed for any association with the risk of hepatotoxicity after IPT commencement. In this model the association between age and the risk of developing hepatotoxicity after commencing IPT was not statistically significant $(p=0.313)$. Concomitant use of nevirapine was the only factor that showed a statistically significant association with hepatotoxicity [relative risk (RR): 2.7; confidence interval (CI): $1.2-6.3, p=0.012]$. Figure 2 shows a Kaplan-Meier estimate of the development of grade 3 or 4 elevation in ALT during IPT.

\section{Discussion}

We report a high incidence rate of severe hepatotoxicity (grade 3 or grade 4 ) of 31.5 per 100 person-years in a cohort of paediatric and adolescent HIV-infected patients on ART. This is in sharp contrast with several studies that have reported little risk of hepatotoxicity, ranging from 0.07 to $1.9 \%[5,13,18]$. However, there are some differences between our study and those that reported lower incidence rates of hepatotoxicity. Grant et al. reported an incidence of $0.07 \%$ clinical hepatotoxicity among predominantly adult, male (95.2\% men, median age of 40 years) South African gold miners [5]. The population under study by Grant et al. was not exclusively HIV infected, and consequently these differences could have affected the different findings. Tedla et al. reported a $1.9 \%$ incidence of severe hepatitis after commencing IPT [13]. However, only $48 \%$ of the participants initiated ART in comparison with our cohort, where all participants were receiving ART.

Our study was conducted in a paediatric and adolescent population where the median age was 10.0 (IQR 7-12) years. Concurrent administration of IPT with nevirapine in HIV-infected patients was significantly associated with hepatotoxicity (RR 2.7; CI $1.2-6.3 ; p=0.012$ ). Whilst this result does not explain the mechanism of how the hepatotoxicity occurs, the association with concomitant administration of IPT whilst a patient is receiving nevirapine is quite evident. In Kenya, the use of IPT among HIV-infected children was evaluated in three HIV care clinics [19]. In their retrospective record review, Masini et al. reported that $0.3 \%$ of HIV-infected children developed adverse drug reactions after IPT commencement [19]. However, identification of adverse drug reactions was based on a health provider inquiry of symptoms and patient/guardian self-reporting of symptoms without any laboratory tests being done. In our study, there was laboratory evaluation of hepatic injury in all patients through ALT measurement. Consequently, patients with liver damage that was not clinically evident were identified.

The safety of IPT has also been evaluated in a prospective double-blind placebo-controlled trial of isoniazid versus placebo given with co-trimoxazole either daily or three times a week in children with HIV in two centres in Cape Town, South Africa [10]. The incidence of grade 3 or 4 toxicity was $4 \%$ in the group that received isoniazid. (This study, like ours, also evaluated ALT). However, the results from the study by Zar et al. [10] were predominantly in children who did not have access to ART. Consequently, the association we found between hepatotoxicity 
and administration of IPT in patients receiving nevirapine-containing ART might not have been apparent in their study. In this study an incidence rate of $31.5 / 100$ personyears (CI: 20.9-45.5) was determined. This rate is higher than previously reported studies in settings similar to those in Zimbabwe [10, 19]. Further pharmacokinetic studies need to be conducted in patients concurrently receiving nevirapine and isoniazid.

A potential limitation for this study was the inability to effectively evaluate adherence to the medicines under study. Adherence assessment to INH and to ARVs was conducted through self-reports and pill counts during routine visits. These two adherence assessment techniques have limitations in evaluating optimum adherence [20]. This has the potential to affect outcomes in participants were the effect of the medicines was not seen. Non-adherence could lead to a larger percentage of patients not experiencing any toxicity effects due to reduced exposure to the medicines. A second limitation was the unavailability of complete ALT laboratory data for 113 participants. The exclusion of these patients from assessment could have introduced a selection bias to the study.

\section{Conclusion}

The incidence of IPT-associated liver enzyme elevations was high in this paediatric and adolescent population. We recommend vigilant monitoring of liver enzymes for patients receiving IPT especially in patients concomitantly receiving nevirapine.

Acknowledgements We would like to thank the Newlands Clinic patients whose data were analysed and the staff who attended to the patients, as well as the Ruedi Luethy Foundation, which financed the work of Newlands Clinic.

Author contributions TinM, TS, CC, SB and MP were responsible for conceptualization of the study. TinM and TilM were responsible for data collection. TS, CC and TilM were responsible for data analysis. TinM and MP provided leadership for the project. TS and TinM provided technical expertise. MP, SB and CC provided clinical expertise for the project. All authors contributed to writing the manuscript and approved the final version for publication.

\section{Compliance with ethical standards}

Funding The authors received no specific funding for this work.

Competing interests Tinashe Mudzviti, Tinei Shamu, Cleophas Chimbetete, Tilda Munengerwa, Sandra Bote and Margaret Pascoe declare that no competing interests exist for the work undertaken.

Open Access This article is distributed under the terms of the Creative Commons Attribution-NonCommercial 4.0 International License (http://creativecommons.org/licenses/by-nc/4.0/), which permits any noncommercial use, distribution, and reproduction in any medium, provided you give appropriate credit to the original author(s) and the source, provide a link to the Creative Commons license, and indicate if changes were made.

\section{References}

1. World Health Organization. Global tuberculosis report. Geneva: WHO Library cataloguing-in-publication data; 2014.

2. Rose DN, Schechter CB, Silver AL. The age threshold for isoniazid chemoprophylaxis A decision analysis for low-risk tuberculin reactors. JAMA. 1986;256(19):2709-13.

3. Durovni B, Cavalcante SC, Saraceni V, Vellozo V, Israel G, King BS, et al. The implementation of isoniazid preventive therapy in HIV clinics: the experience from the TB/HIV in Rio (THRio) study. AIDS. 2010;24(Suppl 5):S49-56. https://doi. org/10.1097/01.aids.0000391022.95412.a6.

4. Akolo C, Bada F, Okpokoro E, Nwanne O, Iziduh S, Usoroh E, et al. Debunking the myths perpetuating low implementation of isoniazid preventive therapy amongst human immunodeficiency virus-infected persons. World J Virol. 2015;4(2):105-12. https:// doi.org/10.5501/wjv.v4.i2.105.

5. Grant AD, Mngadi KT, van Halsema CL, Luttig MM, Fielding KL, Churchyard GJ. Adverse events with isoniazid preventive therapy: experience from a large trial. AIDS. 2010;24(Suppl 5):S29-36. https://doi.org/10.1097/01.aids.0000391019.10661.66.

6. Madhi SA, Nachman S, Violari A, Kim S, Cotton MF, Bobat R, et al. Primary isoniazid prophylaxis against tuberculosis in HIVexposed children. N Engl J Med. 2011;365(1):21-31. https://doi. org/10.1056/NEJMoa1011214.

7. Le Roux SM, Cotton MF, Myer L, le Roux DM, Schaaf HS, Lombard CJ, et al. Safety of long-term isoniazid preventive therapy in children with HIV: a comparison of two dosing schedules. Int $\mathrm{J}$ Tuberc Lung Dis. 2013;17(1):26-31. https://doi.org/10.5588/ijtld .11 .0820 .

8. Takarinda KC, Choto RC, Harries AD, Mutasa-Apollo T, Chakanyuka-Musabhu C. Routine implementation of isoniazid preventive therapy in HIV-infected patients in seven pilot sites in Zimbabwe. Public Health Action. 2017;7(1):55-60.

9. Zunza M, Gray DM, Young T, Cotton M, Zar HJ. Isoniazid for preventing tuberculosis in HIV-infected children. Cochrane Database Syst Rev. 2017;8:CD006418. https://doi.org/10.1002/14651 858.cd006418.pub3.

10. Zar HJ, Cotton MF, Strauss S, Karpakis J, Hussey G, Schaaf HS, et al. Effect of isoniazid prophylaxis on mortality and incidence of tuberculosis in children with HIV: randomised controlled trial. BMJ. 2007;334(7585):136. https://doi.org/10.1136/bmj.39000 .486400 .55 .

11. Mataranyika PA, Kibuule D, Kalemeera F, Kaura H, Godman B, Rennie TW. Liver enzyme elevations in a cohort of HIV/AIDS patients on first-lineantiretroviral therapy in Namibia: findings and implications. Alexandria J Med. 2018;54:49-56.

12. Samandari T, Agizew TB, Nyirenda S, Tedla Z, Sibanda T, Shang N, et al. 6-month versus 36-month isoniazid preventive treatment for tuberculosis in adults with HIV infection in Botswana: a randomised, double-blind, placebo-controlled trial. Lancet. 2011;377(9777):1588-98. https://doi.org/10.1016/S0140 -6736(11)60204-3.

13. Tedla Z, Nyirenda S, Peeler C, Agizew T, Sibanda T, Motsamai O, et al. Isoniazid-associated hepatitis and antiretroviral drugs during tuberculosis prophylaxis in hiv-infected adults in Botswana. 
Am J Respir Crit Care Med. 2010;182(2):278-85. https://doi. org/10.1164/rccm.200911-1783OC.

14. Decloedt EH, Mwansa-Kambafwile J, van der Walt JS, McIlleron H, Denti P, Smith P, et al. The pharmacokinetics of nevirapine when given with isoniazid in South African HIV-infected individuals. Int J Tuberc Lung Dis. 2013;17(3):333-5. https://doi. org/10.5588/ijtld.12.0427.

15. Shamu T, Chimbetete C, Shawarira-Bote S, Mudzviti T, Luthy R. Outcomes of an HIV cohort after a decade of comprehensive care at Newlands Clinic in Harare, Zimbabwe: TENART cohort. PLoS One. 2017;12(10):e0186726. https://doi.org/10.1371/journ al.pone. 0186726.

16. Tweed CD, Wills GH, Crook AM, Dawson R, Diacon AH, Louw $\mathrm{CE}$, et al. Liver toxicity associated with tuberculosis chemotherapy in the REMoxTB study. BMC Med. 2018;16(1):46. https:// doi.org/10.1186/s12916-018-1033-7.

17. Ministry of Health and Child Care Zimbabwe. Guidelines for antiretroviral therapy for the prevention and treatment of HIV in Zimbabwe, 2013. In: Directorate TAaT, editor. 2013.
18. Rangaka MX, Wilkinson RJ, Boulle A, Glynn JR, Fielding K, van Cutsem $\mathrm{G}$, et al. Isoniazid plus antiretroviral therapy to prevent tuberculosis: a randomised double-blind, placebo-controlled trial. Lancet. 2014;384(9944):682-90. https://doi.org/10.1016/S0140 -6736(14)60162-8.

19. Masini EO, Sitienei J, Weyeinga H. Outcomes of isoniazid prophylaxis among HIV-infected children attending routine HIV care in Kenya. Public Health Action. 2013;3(3):204-8. https://doi. org/10.5588/pha.13.0013.

20. Mudzviti T, Mandizvidza T, Ngara B, Chimbetete C, Maponga CC, Morse GD. Pill dumping in adolescents receiving a boosted protease inhibitor regimen as part of second-line antiretroviral therapy: experiences from an urban HIV clinic. World STI \& HIV Congress; Brisbane Australia: Sexually Transmitted Infections; 2015. p. A33.3-A4. 